\title{
Efeito da Terapia Comunitária Integrativa sobre os sintomas de ansiedade em adolescentes no contexto escolar
}

\author{
Effect of Integrative Community Therapy on anxiety symptoms in adolescents in the school context \\ Efecto de la Terapia Comunitaria Integradora sobre los síntomas de ansiedad en adolescentes en el \\ contexto escolar
}

Recebido: 15/02/2021 | Revisado: 21/02/2021 | Aceito: 25/02/2021 | Publicado: 04/03/2021

\author{
Mariane Inaraí Alves \\ ORCID: https://orcid.org/0000-0001-6792-1398 \\ Universidade Federal de Alfenas, Brasil \\ E-mail: mariane.alves@unifal-mg.edu.br \\ Adriana Olimpia Barbosa Felipe \\ ORCID: https://orcid.org/0000-0002-4491-5750 \\ Universidade Federal de Alfenas, Brasil \\ E-mail: adriana.felipe@unifal-mg.edu.br \\ Vânia Regina Bressan \\ ORCID: https://orcid.org/0000-0003-2227-2755 \\ Universidade Federal de Alfenas, Brasil \\ E-mail: vania.bressan@unifal-mg.edu.br \\ Zélia Marilda Rodrigues Resck \\ ORCID: https://orcid.org/0000-0002-3752-8381 \\ Universidade Federal de Alfenas, Brasil \\ E-mail: zelia.resk@unifal-mg.edu.br \\ Denis da Silva Moreira \\ ORCID: https://orcid.org/0000-0002-5055-5210 \\ Universidade Federal de Alfenas, Brasil \\ E-mail: denis.moreira@unifal-mg.edu.br
}

\begin{abstract}
Resumo
Objetivo: Avaliar o efeito da Terapia Comunitária Integrativa sobre os sintomas de ansiedade em adolescentes no contexto escolar. Metodologia: Trata-se de uma pesquisa com abordagem metodológica quantitativa, quase experimental do tipo pré e pós-teste, desenvolvida com 56 adolescentes escolares. Os instrumentos para a coleta de dados foram: Caracterização dos participantes, Classificação Econômica e o Screen for Child Anxiety Related Emotional Disorders. O Screen for Child Anxiety Related Emotional Disorders foi aplicado pré e pós-intervenção. Realizou-se um total de cinco sessões de Terapia Comunitária Integrativa semanalmente na escola. Para análise dos dados utilizou-se estatística descritiva, testes de associação, análise de variância e comparação de médias. Resultados: Cerca de 76,8\% dos adolescentes apresentaram sintomas ansiosos. As meninas tiveram maiores escores de ansiedade e apresentaram maiores chances (Odds ratio:9,8) para desenvolverem sintomas ansiosos do que os meninos. Os escores de ansiedade tiveram uma redução significativa após as cinco sessões de Terapia Comunitária Integrativa na amostra total (média pré-intervenção: 48,2; média pós-intervenção: 42,6) e na amostra dos adolescentes com escores acima do ponto de corte (média pré-intervenção= 56,6; média pós-intervenção= 49,0). Conclusão: A Terapia Comunitária Integrativa foi uma intervenção efetiva na diminuição dos escores de ansiedade, sendo uma importante estratégia de apoio psicossocial que pode ser utilizada pelo enfermeiro na prevenção do sofrimento psicoemocional dos adolescentes. Palavras-chave: Enfermagem; Terapias complementares; Adolescente; Ansiedade; Saúde mental; Serviços de saúde mental escolar.
\end{abstract}

\begin{abstract}
Objective: To evaluate the effect of Integrative Community Therapy on anxiety symptoms in adolescents in the school context. Methodology: It is a research with a quantitative methodological approach using a pre-and post-test evaluation developed with 56 school adolescents. The instruments for data collection were: Characterization of Participants, Economic Classification and the Screen for Child Anxiety Related Emotional Disorders. Screen for Child Anxiety Related Emotional Disorders was applied pre- and post-intervention. A total of five sessions of Integrative Community Therapy were held weekly at the school. For data analysis, descriptive statistics, association tests, analysis of variance and comparison of means were used. Results: About $76.8 \%$ of the adolescents had anxious symptoms. Girls had higher anxiety scores and were more likely (Odds ratio: 9.8 ) to develop anxiety symptoms than boys. Anxiety scores had a significant reduction after five sessions of Integrative Community Therapy for both total sample (pre-intervention mean:
\end{abstract}


48.2; post-intervention mean: 42.6) and for those adolescents scoring above the cut-off point (pre-intervention mean: 56.6; post-intervention mean: 49.0). Conclusion: Integrative Community Therapy was an effective intervention in decreasing anxiety scores, being an important psychosocial support strategy that can be used by nurses to prevent adolescents suffering from psycho-emotional disorders.

Keywords: Nursing; Complementary therapies; Adolescent; Anxiety; Mental health; School mental health services.

\section{Resumen}

Objetivo: Evaluar el efecto de la Terapia Comunitaria Integradora sobre los síntomas de ansiedad en adolescentes en el contexto escolar. Metodología: Se trata de una investigación con enfoque metodológico cuantitativo cuasi-experimental del tipo pre y post-test, desarrollada con 56 adolescentes escolares. Los instrumentos para la recolección de datos fueron: Caracterización de Participantes, Clasificación Económica y Screen for Child Anxiety Related Emotional Disorders. Se aplicó Screen for Child Anxiety Related Emotional Disorders antes y después de la intervención. Se llevaron a cabo un total de cinco sesiones de Terapia Comunitaria Integradora semanalmente en la escuela. Para el análisis de los datos se utilizó estadística descriptiva, pruebas de asociación, análisis de varianza y comparación de medias. Resultados: Aproximadamente el 76,8\% de los adolescentes presentaban síntomas de ansiedad. Las niñas tenían puntuaciones de ansiedad más altas y tenían más probabilidades (razón de probabilidades: 9,8) de desarrollar síntomas de ansiedad que los niños. Las puntuaciones de ansiedad tuvieron una reducción significativa después de las cinco sesiones de Terapia Comunitaria Integrativa en la muestra total (media preintervención: 48,2; media posintervención: 42,6) y en la muestra de adolescentes con puntuaciones superiores al punto de corte (media preintervención: 56,6; promedio posintervención: 49,0). Conclusión: La Terapia Comunitaria Integradora fue una intervención eficaz para disminuir los puntajes de ansiedad, siendo una importante estrategia de apoyo psicosocial que pueden utilizar las enfermeras para prevenir el sufrimiento psicoemocional de los adolescentes.

Palabras clave: Enfermería; Terapias complementarias; Adolescente; Ansiedad; Salud mental; Servicios de salud mental escolar.

\section{Introdução}

A adolescência é um período de intensas mudanças a nível biológico, psicológico ou social e uma fase determinante para o desenvolvimento e a manutenção de hábitos sociais e emocionais importantes para o bem-estar mental. Entre eles estão o desenvolvimento da capacidade de enfrentamento e resolução de problemas, das habilidades interpessoais, de aprender administrar as emoções e ter ambientes de apoio na família, na escola e na comunidade (Organização Pan-Americana da Saúde, 2018).

A ansiedade tem seu início marcado na infância e na pré-adolescência (Organização Pan-Americana da Saúde, 2018) e está entre as principais necessidades de cuidado em saúde mental na adolescência (Erse et al., 2016). Em todo o mundo, a ansiedade é a $8^{\circ}$ causa de doença e incapacidade entre os adolescentes o que pode interferir negativamente no seu desenvolvimento psicossocial (Organização Pan-Americana da Saúde, 2018).

Neste sentido, tanto os serviços de saúde como a escola devem instituir intervenções de prevenção e promoção de saúde mental dos adolescentes (Cid et al., 2019; Erse et al., 2016; Nunes et al., 2020; Soares et al., 2020). Essas intervenções devem estar focadas na potencialização das competências pessoais e individuais, no fortalecimento da autoestima, da capacidade de resolução de problemas e na busca de ajuda, promovendo um espaço psicológico seguro e positivo, e assim diminuir os fatores de risco e fortalecer os fatores protetivos (Erse et al., 2016; Organização Pan-Americana da Saúde, 2018). Torna-se extremamente importante para o desenvolvimento saudável dos adolescentes receberem cuidados e serem compreendidos (Tardivo et al., 2019).

Nesta perspectiva, a Terapia Comunitária Integrativa (TCI) que integra a Política Nacional de Saúde Mental desde 2013 e Política Nacional de Práticas Integrativas e Complementares (PNPIC) desde 2017 (Portaria 849, 2017), vem se consolidando como uma nova tecnologia de cuidado dentro do contexto da saúde mental (Garcia et al., 2018), constituindo-se um instrumento valioso de intervenção psicossocial na saúde pública. A mesma não pretende substituir os outros serviços de saúde, mas complementá-los, de modo a ampliar as ações promocionais e preventivas. A TCI acolhe, escuta e cuida das pessoas e de seus sofrimentos (Barreto, 2019), e contribui para o empoderamento, o sentimento de pertença (Moura et al., 2017), de ressignificação e mudança da situação que gera angústia (Carneiro et al., 2020).

Complementa-se também que esta estratégia facilita o resgate da autoestima, fortalece a capacidade do indivíduo em 
lidar com os problemas e se adaptar as mudanças, estimula sua autoconfiança, uma vez que fortalece recursos individuais e coletivos. É um instrumento de construção de redes de apoio social que possibilita a criação de vínculos e a formação de um ambiente propício para trocas de experiências e de resgate das habilidades de superação por meio da formação de recursos sócios emocionais (Barreto, 2019; Oliveira et al., 2017).

Portanto, torna-se necessário compreender a Terapia Comunitária Integrativa e sua atuação sobre os sintomas de ansiedade com foco na população adolescente no contexto escolar e fundamentar-se em dados importantes para uma atuação efetiva dos profissionais de saúde e educação. Sendo assim, esta estratégia poderá ser implementada no contexto escolar a fim de prevenir e tratar precocemente os sofrimentos psicoemocionais, que compromete a qualidade de vida dessa população.

Diante do exposto, este estudo objetivou avaliar o efeito da Terapia Comunitária Integrativa sobre os sintomas de ansiedade em adolescentes no contexto escolar.

\section{Metodologia}

\subsection{Tipo de estudo}

Trata-se de uma pesquisa com abordagem metodológica quantitativa (Pereira et al., 2018), quase experimental, sem grupo controle do tipo pré e pós-teste. A pesquisa quase experimental envolve uma intervenção e é realizada para estudos quantitativos na qual é feita uma comparação pré e pós-intervenção com apenas um grupo (Polit \& Beck, 2018).

\subsection{Local de estudo}

O estudo foi realizado em uma escola estadual pública de um município do sul de Minas Gerais, MG, Brasil.

\subsection{Período de coleta de dados}

A coleta de dados ocorreu no período de outubro a novembro de 2019.

\subsection{Participantes do estudo}

A TCI é uma estratégia psicoemocional que sua participação depende do desejo de estar presente, compartilhar suas histórias de vida e manifestar de maneira empática seus sentimentos, assim de acordo com Boaretto et al. (2020) a amostragem probabilística não intencional não se aplica nesta investigação, sendo realizada a amostragem por conveniência.

Foram estabelecidos como critérios de inclusão: idade entre 12 e 17 anos, ser alfabetizado, estar regularmente matriculado na escola e ter participado de no mínimo 60\% das rodas de Terapia Comunitária Integrativa conduzidas na escola em estudo. Para os critérios de exclusão, foram considerados: ter participado anteriormente de sessões de TCI ou qualquer outra terapia três meses anteriores à intervenção, apresentar condições neurológicas e cognitivas que impossibilitem o adolescente de responder os instrumentos. Portanto, a amostra do estudo foi constituída por 56 adolescentes

\subsection{Instrumentos de coleta de dados}

Foram utilizados três instrumentos autoaplicáveis para a realização da pesquisa.

Para a caracterização dos adolescentes, utilizou-se 13 questões relevantes do questionário elaborado por Tavares, Béria e Lima (Tavares et al., 2004) e acrescida duas perguntas: de identificação do participante e a participação em terapias prévias. Utilizou-se também o Critério de Classificação Econômica Brasil, que analisa a posse de itens de consumo, instrução do chefe e acesso a serviços públicos, com a somatória de todos os itens, a pontuação varia de 0 a 100. Classificados de acordo com os pontos de corte: A - 45 a 100 pontos; B1 - 38 a 44; B2 - 29 a 37; C1 -23 a 28; C2 - 27 a 17; D-E - 0 a 16 (Associação Brasileira 
de empresas de Pesquisa, 2016).

Para a avaliação e triagem dos sintomas de ansiedade utilizou-se o Screen for Child Anxiety Related Emotional Disorders (SCARED) (Barbosa \& Barbosa, 2001), foi traduzido e validado para a população brasileira em 2002. O questionário é constituído de 38 itens que avalia a ansiedade na população de 7 a 17 anos. O ponto de corte é de 34 pontos, o adolescente com pontuação igual ou superior ao ponto de corte apresenta sintomas de ansiedade (Barbosa et al., 2002).

\subsection{Procedimento para a coleta de dados}

No primeiro contato, os adolescentes foram esclarecidos quantos aos objetivos, aos métodos da pesquisa, sobre a condução das rodas de TCI, e entregue o Termo de Assentimento para a sua assinatura. Solicitou-se também que os mesmos entregassem aos pais e responsáveis o Termo de Consentimento Livre e Esclarecido para a assinatura dos pais e autorização para a participação na pesquisa.

As coletas foram realizadas pela pesquisadora responsável. Os instrumentos de coletas de dados (autoaplicáveis) foram coletados em 2 momentos: A primeira coleta foi realizada antes da intervenção por meio da aplicação do questionário de Caracterização dos Participantes e o SCARED (pré-teste). No segundo momento, após a realização da intervenção (cinco rodas de TCI), foram aplicados novamente o SCARED (pós-teste).

\subsection{Intervenção}

Para a condução das rodas de TCI a pesquisadora responsável participou de um curso de formação de Terapeutas Comunitários.

A realização da intervenção com os adolescentes consistiu em um total de cinco sessões com cada grupo, uma vez na semana com duração média de uma hora e meia conforme preconizado por Barreto (Barreto, 2019) e evidenciado em outros estudos (Brum \& Teixeira, 2020; Lutterbach, 2017). Os participantes foram divididos em três grupos, cada grupo foi composto por um número aproximado de 20 adolescentes. As rodas de TCI foram realizadas em uma sala de aula preestabelecida pela direção da instituição de ensino, que possibilitou a privacidade dos participantes e a execução da intervenção. As mesmas foram conduzidas pela pesquisadora e por outros terapeutas comunitários os quais seguiram um protocolo pré-definido com as cinco etapas preconizadas por Barreto (Barreto, 2019).

Acolhimento - Nesta etapa o terapeuta comunitário dava boas-vindas ao grupo pedia para sentar em círculo para ficarem confortáveis e solicitava que os participantes se apresentassem. Uma música era cantada para deixar o clima leve entre os participantes. Após esse momento era apresentado uma síntese do que é a Terapia Comunitária e era explicado sobre as regras (respeitar quem está falando, fazer silêncio, falar da própria história, utilizando a $1^{\circ}$ pessoa do singular (eu), cuidar para não dar conselhos, discursar ou dar sermões, utilizar músicas que tenham a ver com o tema escolhido, bem como piadas, histórias e provérbios relacionados). A cada sessão era proposto uma dinâmica de grupo com intuito de interação, resgate da autoestima, estimular o empoderamento e fortalecimento dos laços de amizade;

Escolha do tema - Na escolha do tema a fala ficava aberta para os participantes falarem de seus sofrimentos, angústia, tristeza vivenciada no seu cotidiano. Após a exposição dos problemas, eram identificados os temas e o grupo votava naquele que mais se identificava;

Contextualização - Nesta fase a pessoa cujo tema era escolhido falava sobre o problema ou situação apresentada. Após ela descrever a situação, era aberto para todos fazerem perguntas para esclarecer e entender melhor a questão. Então o terapeuta extraia dos depoimentos da pessoa escolhida uma pergunta chave que permitia a reflexão do grupo - o mote;

Problematização - Neste momento o mote era conduzido para o grupo e os adolescentes relatavam sobre sua própria experiência em relação ao sentimento vivenciado, as estratégias que fez para superar ou se ainda estava superando; 
Encerramento - No final da roda de TCI o terapeuta fez rituais de agregação e conotações positivas, permitindo que o grupo pudesse refletir, aprender com a troca de experiências reforçando a autoestima e estratégias de enfrentamento. Sempre era aberto um espaço para os participantes falarem sobre o que aprenderam e o que estavam levando do momento partilhado durante a roda. Todas as sessões de TCI terminaram com os agradecimentos dos terapeutas e com um convite para os próximos encontros.

\subsection{Tratamento e análise dos dados}

Foram realizadas análises estatísticas utilizando o software Bioestat, versão 5.0.

Os dados foram analisados a partir da estatística descritiva com análise de distribuições e frequência. $\mathrm{O}$ teste de D’Agostino-Pearson verificou que a variável ansiedade no momento pré e pós-intervenção apresentou distribuição normal. A variável classe econômica e com quem vive o adolescente não seguiu distribuição normal. Dessa forma, os dados que seguiram distribuição normal foram tratados com a estatística paramétrica e os que não seguiram com a estatística não paramétrica.

Foi aplicado então, o teste de t para amostras relacionadas na comparação da variável ansiedade no momento pré e pósintervenção.

Para verificar a relação entre ansiedade e características sociodemográficas, utilizou-se o teste t para amostras independentes. Somente para as variáveis que não seguiram distribuição normal, classe econômica e com quem vive o adolescente, foram aplicados os testes de Mann-Whitney e o teste de Kruskal Wallis, respectivamente. Para análise da razão de chance foi utilizado o Odss Ratio. O nível de significância estabelecido foi de 5\%.

\subsection{Aspectos éticos}

Após anuência institucional da escola para a realização da pesquisa, o projeto foi submetido para apreciação e aprovação do Comitê de Ética de acordo com as Diretrizes e Normas Regulamentadoras de Pesquisa envolvendo seres Humanos, Resolução do Conselho Nacional de Saúde 466/12 (Conselho Nacional de Saúde, 2012) e recebeu parecer favorável no 3.509.507.

Somente participaram da pesquisa aqueles que foram autorizados pelos pais ou responsáveis mediante assinatura do Termo de Consentimento Livre e Esclarecido e aceitaram participar da pesquisa por meio da assinatura do Termo de Assentimento. Foram garantidos o anonimato e o sigilo das informações prestadas pelos respondentes.

\section{Resultados}

Os participantes do estudo foram constituídos por 56 adolescentes, sendo 14 (30,4\%) do sexo masculino e 39 (69,6\%) do sexo feminino, com média de idade de 15 anos, 38 (67,8\%) estavam matriculados no ensino médio, 30 (53,6\%) foram classificados economicamente entre as classes B2 e C1 e $54(96,4 \%)$ relataram ser solteiros.

Os adolescentes referenciaram que $34(60,7 \%)$ dos pais não vivem juntos, mas $25(73,5 \%)$ reportaram que seus pais já viveram juntos e se separaram, 24 (42,9\%) moravam com os pais e $41(73,2 \%)$ não exerciam atividade laboral.

Quanto à religiosidade $32(57,1 \%)$ referiram ser católicos e 18 (33,9\%) evangélicos, 41 (73,2\%) relataram praticar religião, contudo 34 (60,7\%) não participam de grupos religiosos, $55(98,2 \%)$ acreditam em Deus e $46(82,1 \%)$ costumam rezar ou orar frente a um problema.

Na avaliação dos escores de ansiedade, 43 (76,8\%) apresentaram sintomas de ansiedade (escores de ansiedade acima de 34 pontos).

Na Tabela 1 está apresentada a análise da associação entre as variáveis sociodemográficas com a ansiedade. 
Tabela 1 - Relação entre as variáveis sociodemográficas com os escores de ansiedade dos adolescentes (n=56) em uma escola de um município do sul de Minas Gerais, MG, 2019.

\begin{tabular}{|c|c|c|c|c|c|c|}
\hline \multirow[b]{2}{*}{ Variáveis sociodemográficas } & \multicolumn{4}{|c|}{ Escores de ansiedade } & \multirow[b]{2}{*}{ Mínimo } & \multirow[b]{2}{*}{ valor-p } \\
\hline & Média & Mediana & DP & Máximo & & \\
\hline \multicolumn{7}{|l|}{ Sexo } \\
\hline Masculino & 36,5 & 27,0 & 22,3 & 89 & 11 & \multirow{2}{*}{$0,0022 *$} \\
\hline Feminino & 53,3 & 53,0 & 15,7 & 85 & 19 & \\
\hline \multicolumn{7}{|l|}{ Faixa etária } \\
\hline 12 a 14 anos & 53,2 & 52,0 & 21,2 & 85 & 11 & \multirow[b]{2}{*}{$0,2077 *$} \\
\hline 15 a 17 anos & 45,9 & 52,5 & 18,4 & 89 & 19 & \\
\hline \multicolumn{7}{|l|}{ Pais vivem juntos } \\
\hline $\operatorname{Sim}$ & 47,3 & 51,5 & 24,6 & 89 & 11 & \multirow{2}{*}{$0,7877 *$} \\
\hline Não & 48,8 & 52,5 & 16,1 & 81 & 13 & \\
\hline \multicolumn{7}{|l|}{ Com quem vive } \\
\hline Pai e mãe & 45,8 & 49,0 & 24,0 & 89 & 11 & \\
\hline Pai ou mãe & 47,8 & 51,0 & 16,3 & 74 & 13 & \\
\hline Outros & 54,5 & 53,0 & 11,1 & 81 & 40 & $0,6447 \dagger$ \\
\hline \multicolumn{7}{|l|}{ Trabalha } \\
\hline Sim & 47,7 & 46,0 & 23,8 & 89 & 11 & \multirow{2}{*}{$0,9150^{*}$} \\
\hline Não & 48,4 & 52,0 & 17,8 & 85 & 13 & \\
\hline \multicolumn{7}{|l|}{ Filiação Religiosa } \\
\hline Sim & 48,2 & 51,5 & 19,8 & 89 & 11 & \multirow{2}{*}{$0,9834 *$} \\
\hline Não & 48,0 & 53,5 & 13,5 & 57 & 28 & \\
\hline \multicolumn{7}{|l|}{ Pratica de religião } \\
\hline $\operatorname{Sim}$ & 48,5 & 51,0 & 20,6 & 89 & 11 & \multirow[b]{2}{*}{$0,8423 *$} \\
\hline Não & 47,3 & 53,0 & 15,9 & 67 & 14 & \\
\hline \multicolumn{7}{|l|}{ Participa de grupo religioso } \\
\hline $\operatorname{Sim}$ & 54,7 & 53,5 & 18,3 & 89 & 19 & \multirow{2}{*}{$0,0427 *$} \\
\hline Não & 44,0 & 46,0 & 19,1 & 81 & 11 & \\
\hline \multicolumn{7}{|l|}{ Rezar ou orar } \\
\hline Sim & 49,9 & 53,0 & 18,1 & 85 & 11 & \multirow{2}{*}{$0,1514 *$} \\
\hline Não & 40,2 & 39,0 & 24,0 & 89 & 13 & \\
\hline \multicolumn{7}{|l|}{ Classe Econômica } \\
\hline A e B & 44,9 & 46,0 & 21,3 & 89 & 11 & \multirow{2}{*}{$0,2370 \ddagger$} \\
\hline$C, D$ e E & 51,1 & 53,0 & 17,4 & 85 & 16 & \\
\hline
\end{tabular}

Fonte: Autores. Aplicação *teste t; † teste Kruskal Wallis, łteste Mann Whitney

$\mathrm{Na}$ análise entre as variáveis sociodemográficas com a ansiedade constatou-se associação estatisticamente significante com o sexo feminino. As meninas apresentaram média dos escores de ansiedade superior quando comparado com os meninos.

Foi observado também que a variável ansiedade teve uma associação com a participação em grupos religiosos. Aqueles adolescentes que não participaram de grupos ligados à religião tiveram média dos escores de ansiedade inferiores quando comparados com os adolescentes que frequentaram grupos religiosos.

Na avaliação pelo Odds ratio ( $\mathrm{p}=0,0017$ ) foi constatado que os adolescentes do sexo feminino tiveram 9,8 mais chances de desenvolver sintomas de ansiedade.

Para a associação entre a ansiedade e a variável participação em grupos religiosos pela razão de chance não foi observado significância estatística.

Na Tabela 2 encontram-se os resultados referentes aos escores de ansiedade pré e pós-intervenção (TCI). 
Tabela 2 - Análise dos resultados dos escores de ansiedade dos adolescentes no momento pré e pós-intervenção em uma escola de um município do sul de Minas Gerais, MG, 2019.

\begin{tabular}{cccccc}
\hline Momento & Variável & Média & Mediana & Desvio Padrão & valor- $\boldsymbol{p}$ \\
\hline Pré & Ansiedade & 48,2 & 52,0 & 19,4 & \\
\multirow{2}{*}{ Pós } & Ansiedade & 42,6 & 43,0 & 20,2 & $0,0023^{*}$ \\
\hline \multirow{5}{*}{ Pré } & Escore Geral $(\mathbf{n}=\mathbf{5 6})$ & \\
Pós & Ansiedade & 56,6 & 54,0 & 13,0 & \multirow{2}{*}{$0,0005^{*}$} \\
\hline
\end{tabular}

Fonte: Autores. p: nível de significância; *test t

Ao aplicar o teste t observou-se uma diferença significativa nos escores de ansiedade quando comparados as médias nos momentos pré e pós-intervenção, tanto para a amostra total, quanto para aqueles que tiveram ponto de corte superior a 34 .

Na Tabela 3 serão apresentadas as análises referentes aos escores de ansiedade no momento pré e pós-intervenção por sexo.

Tabela 3 - Análise dos resultados dos escores de ansiedade no momento pré e pós-intervenção por sexo em uma escola de um município do sul de Minas Gerais, MG, 2019.

\begin{tabular}{ccccccc}
\hline Sexo & Momento & Variável & Média & Mediana & Desvio Padrão & valor-p \\
\hline Feminino & Pré & Ansiedade & 53,3 & 53,0 & 15,7 & $0,0007^{*}$ \\
& Pós & Ansiedade & 46,4 & 47,0 & 20,1 & \\
\multirow{2}{*}{ Masculino } & Pré & Ansiedade & 36,5 & 27,0 & 22,3 & $0,5041^{*}$ \\
& Pós & Ansiedade & 33,9 & 32,0 & 18,2 & \\
\hline
\end{tabular}

Fonte: Autores. p: nível de significância; *test t

Evidenciou-se uma diferença significativa na diminuição dos escores de ansiedade dos adolescentes do sexo feminino. Os adolescentes do sexo masculino não apresentaram uma redução significativa na média dos escores quando comparados pré e pós-intervenção

\section{Discussão}

$\mathrm{Na}$ avaliação da ansiedade entre os adolescentes verificou-se que 76,8\% apresentaram sintomas de ansiedade, frequência superior ao encontrado na literatura.

Em uma avaliação realizada com 678 adolescentes da Índia, por meio da triagem com o SCARED, a percentual de transtornos de ansiedade foi de 16,6\% (Madasu et al., 2019).

Em outro estudo que também utilizou o SCARED para a triagem de 1092 jovens ( 7 a 18 anos) com o objetivo de examinar os transtornos de ansiedade pediátrica no Instituto Nacional de Saúde Mental nos EUA, constatou que 45,8\% procuravam tratamento para um transtorno de ansiedade (Behrens et al., 2019). 
Pesquisa realizada no Canadá buscou compreender as tendências na prevalência de doenças mentais em jovens de 12 a 24 anos entre os anos de 2011 a 2018 encontrou um aumento na prevalência de saúde mental ruim/razoável percebida e de diagnósticos de transtornos de humor e ansiedade nessa faixa etária ao decorrer dos anos (Wiens et al., 2020).

No Brasil em uma investigação realizada em 6 escolas na cidade de Porto Alegre com 119 estudantes de 9 a 18 verificou através da triagem com o SCARED que 37,0\% dos participantes tiveram pelo menos um diagnóstico de transtorno de ansiedade (Desousa et al., 2013).

O transtorno de ansiedade foi classificado como o sexto maior contribuinte para a perda de saúde não fatal em todo o mundo em 2015 (Organização Mundial da Saúde, 2017) e representa um dos transtornos psiquiátricos mais comuns entre crianças e adolescentes. A deteçãa precoce permite reduzir a ocorrência de mais sintomas negativos, notas baixas, problemas comportamentais e familiares, baixa escolaridade, absenteísmo escolar, depressão e abuso de substâncias psicoativas (Beesdo et al., 2009; Ingul \& Nordahl, 2013; Runyon et al., 2018).

Os sintomas de ansiedade encontrados na presente investigação foram maiores nas meninas quando comparados com os meninos e elas também apresentaram maiores chances para o desenvolvimento desses sintomas.

Esses dados corroboram com a literatura, nos quais estudos comprovam que a ansiedade aumenta na adolescência e principalmente entre mulheres (Gomez \& Vance, 2016; Grolli et al., 2017; Patias et al., 2016). No Canadá foi observado um aumento na prevalência anual de distúrbios de humor e de ansiedade entre os jovens e essas tendências foram mais evidentes para mulheres (Wiens et al., 2020). O sexo feminino está associado a transtorno de ansiedade e as meninas apresentam maiores chances para desenvolvê-lo em comparação aos meninos (Madasu et al., 2019; Orellana et al., 2020).

Os adolescentes que não participavam de grupos ligados à religião tiveram média dos escores de ansiedade inferiores quando comparados com os adolescentes que participavam de grupos religiosos. Não há um consenso na literatura sobre os efeitos da religiosidade/espiritualidade sobre a saúde mental dos adolescentes (Thiengo et al., 2019), o que se torna relevante a condução de novas pesquisas com essas variáveis.

Alguns adolescentes frequentam os serviços religiosos porque muitos se veem pressionados pela família para praticar a religião, o que a arbitrariedade no exercício da crença religiosa pode resultar em se rebelar de alguma forma, por meio de comportamento prejudicial à saúde. Estes conflitos podem gerar sofrimento, ocorrendo uma desordem na regulação de suas emoções (Malinakova et al., 2019), afetando o seu bem-estar psicológico (Zidkova et al., 2020).

Em um estudo realizado nos EUA, aqueles que acreditavam em Deus e apresentaram enfrentamento religioso negativo tiveram níveis mais altos de ansiedade (Kent et al., 2020).

Segundo Zidkova (Zidkova et al., 2020) o simples exercício da religião não está associado a menor chance de queixas de problemas de saúde, enquanto um maior nível de espiritualidade foi associado a menores chances relatadas pelos adolescentes.

Por outro lado, alguns autores revelaram efeitos positivos da religião e espiritualidade sobre a saúde (Inoue \& Vecina, 2017), as condutas dos adolescentes e comportamentos de risco a saúde (Malinakova et al., 2019). A espiritualidade pode promover o desenvolvimento saudável em adolescentes, aumentando sua capacidade de enfrentamento aos problemas, resultados positivos na saúde mental, bem-estar psicológico e desenvolvimento da capacidade de se adaptar as mudanças (Kim \& Esquivel, 2011; Zidkova et al., 2020).

Em uma pesquisa realizada nos Estados Unidos com adolescentes e jovens de 15 a 25 anos apontou que a espiritualidade/religiosidade estava associada a maior auto eficácia nos cuidados de saúde e pode desempenhar um papel de apoio fortalecendo o autocuidado. Esse cuidado não precisava necessariamente ser baseado na fé, o cuidado espiritual pode envolver atenção plena, meditação ou aconselhamento espiritual (Miller et al., 2017).

A espiritualidade e religiosidade estão ligadas à saúde mental, qualidade de vida e bem-estar dos adolescentes e jovens e exercem uma influência positiva na qualidade de vida dos adolescentes, com fortalecimento dos laços sociais, sendo importante 
para proteção, promoção e recuperação da saúde em situações de insegurança, angústia e superação de doenças (Farinha et al., 2018).

Quanto à análise da efetividade da TCI com adolescentes, evidenciou-se uma diminuição dos escores de ansiedade quando comparados as avaliações que foram realizadas pré e pós a prática da Terapia Comunitária Integrativa. Essa diminuição foi significativa tanto para a amostra geral quanto para aqueles adolescentes que tiveram escores acima do ponto de coorte de 34. No entanto, quando realizado a análise por sexo, pré e pós-intervenção, houve uma diferença significativa na diminuição dos escores de ansiedade somente nas meninas.

Estes dados corroboram com outros estudos que abordaram diferentes populações e locais em relação à contribuição da TCI. A TCI apresentou eficácia na diminuição dos sintomas de ansiedade e depressão após três (Brum \& Teixeira, 2020) e cinco (Boaretto et al., 2020) rodas quando realizada com universitários.

Em um estudo que avaliou a efetividade da TCI na diminuição dos sintomas de ansiedade e depressão em dependentes químicos do sexo masculino encontrou efeitos positivos da intervenção na diminuição dos sintomas de ansiedade e depressão após seis rodas de TCI (Lemes et al., 2020). Destaca-se a necessidade de novos estudos mais robustos em relação às questões de gênero, a saúde mental e a contribuição da TCI no âmbito da adolescência.

Vários estudos com diferentes metodologias e amostras evidenciaram a importância da TCI para o cuidado em saúde mental. A TCI tornou-se uma prática integrativa complementar e mais um recurso a ser usado na Atenção Básica (Reis \& Grandesso, 2014), sendo uma importante tecnologia de cuidado dentro do contexto da saúde mental (Garcia et al., 2018), fortalecendo o empoderamento, o sentimento de pertencimento (Moura et al., 2017) e o poder resiliente (Azevedo et al., 2013) atuando na prevenção do sofrimento emocional dos participantes (Mourão et al., 2016). Portanto, a terapia comunitária acolhe os problemas e os sofrimentos emocionais e auxilia na sua ressignificação, estimula à participação, a fala, a construção de relações e um ambiente de confiança propício para criação de estratégias e de recursos para enfrentar as adversidades (Dallalana, 2015).

Segundo Braga et al. (2013) a TCI pode contribuir para a diminuição dos sofrimentos que trazem angústia, por meio dela é possível criar redes de apoio emocional, permitindo o fortalecimento de vínculos e da capacidade individual e grupal dos participantes para se adaptarem de forma positiva as diferentes mudanças, problemas e conflitos sendo uma importante estratégia de promoção à saúde mental e de cuidado. Destaca-se também como um método terapêutico, no qual ocorre a partilha de experiências, problemas, situações difíceis, dificuldades emocionais, vitórias e superações (Gaete \& Gois, 2020).

Vale ressaltar que a adolescência é uma fase em que as emoções estão afloradas e as habilidades de regulação emocional estão ainda em desenvolvimento. Essa dificuldade de regulação emocional dos adolescentes pode interferir negativamente no seu desenvolvimento psicossocial (Turpyn et al., 2015), o que se evidenciou no presente estudo.

Neste sentido, a TCI se destaca como uma estratégia de cuidado que pode servir de apoio emocional na fase da adolescência, fortalece o autoconhecimento, a autonomia e o amor próprio, que são características importantes para auxiliar nas suas escolhas e conquistas (Moura et al., 2012), o que coaduna com achados desta investigação.

Sabe-se que as estratégias focadas na aceitação emocional, no momento presente e que estimulam a reavaliação da situação estressora e a regulação das emoções são eficazes para os transtornos de humor e de ansiedade (Conroy et al., 2019; Hofmann et al., 2012). Aqueles indivíduos com alta flexibilidade e com maiores diversidades de estratégias para regular suas emoções apresentam uma melhor qualidade de vida e um menor sofrimento emocional (Conroy et al., 2019).

Complementa-se que a TCI vem ganhando espaço como política pública no Brasil. A sua metodologia acessível e participativa permite fortalecer os recursos pessoais e culturais - tais como autoconfiança, resgate da autoestima, autonomia, e criação de vínculos, buscando promover uma reorganização das ideias e pensamentos, fortalecendo os seus recursos individuais para o enfrentamento de seus problemas (Oliveira Cezário et al., 2015). 
Destaca-se que o enfermeiro é um profissional capaz de propor atividades que previnam as alterações na saúde mental, uma vez que, o mesmo consegue avaliar e identificar as necessidades e vulnerabilidades dos adolescentes (Nunes et al., 2020; Tomé et al., 2017). Além disso, o enfermeiro é um dos profissionais capaz de agir como interlocutor entre a saúde e a educação (Silva et al., 2016), a literatura tem referenciado que a área de formação dos pesquisadores com maior destaque nos estudos de intervenção da TCI foi a enfermagem (Zem Igeski et al., 2020). Acredita-se que este fato esteja relacionado ao processo de formação deste profissional de saúde, uma vez que um dos pilares da formação é o olhar holístico e a integralidade no cuidado voltado não apenas as questões biomédicas, mas também para os aspectos psicoemocionais do viver humano.

Enfim, cabe ressaltar, que a assistência do enfermeiro direcionada aos adolescentes focam-se em ações de educação em saúde, atividades em grupos, terapias, buscando atividades que fortalecem o relacionamento interpessoal, além de ações de saúde que envolvem a sua a família, seus pares e a escola (Teixeira et al., 2020).

Os resultados desse estudo poderão contribuir para preencher as lacunas referente a aplicação da TCI no contexto do adolescer, além de favorecer a produção de conhecimento no âmbito da saúde mental e para o fortalecimento de políticas públicas voltadas a essa população.

O estudo apresentou algumas limitações que precisam ser apontadas. Primeiramente, a TCI por ser uma prática relativamente recente, ainda é desconhecida pelos professores e funcionários da instituição de ensino, o que dificultou a disponibilização de um horário reservado para a condução da intervenção. Observou-se também a falta de incentivo aos adolescentes para a participação nas rodas de TCI, muitas vezes deixavam de participar para o cumprimento das atividades escolares que eram propostas sem possibilidade de reposição.

Apesar das limitações apresentadas, o estudo ressalta a utilização da TCI como importante recurso interventivo e estratégia de cuidado para a promoção, prevenção e recuperação da saúde mental de adolescentes e um instrumento valioso para a prática da enfermagem, principalmente no que tange a ansiedade no contexto escolar.

\section{Conclusão}

Os sintomas de ansiedade encontrados nesse estudo apresentaram altas taxas, uma vez que 76,8\% dos adolescentes no âmbito escolar apresentaram sintomas de ansiedade, portanto, os participantes dessa pesquisa são bastante vulneráveis aos problemas de saúde mental, podendo interferir de forma negativa nas suas vidas.

A ansiedade está associada com o sexo feminino, as meninas tiveram maiores escores de ansiedade e apresentam maiores chances para o desenvolvimento de sintomas de ansiedade.

A participação em grupos religiosos não foi um fator protetivo para a ansiedade, os adolescentes que participavam de grupos ligados a religião tiveram média dos escores de ansiedade maiores quando comparados com os adolescentes que não participam de grupos religiosos.

Ao se comparar os efeitos da TCI nos escores de ansiedade pré e pós-intervenção, pode-se inferir que a TCI foi uma intervenção efetiva na diminuição dos escores de ansiedade após cinco sessões, uma vez que houve uma redução significativa dos sintomas.

Nessa perspectiva, é essencial a implementação de políticas públicas mais efetivas, que vão além das instituições de saúde, voltadas para a saúde mental de adolescentes no âmbito escolar que é um local onde os adolescentes passam grande parte do seu tempo. São necessárias intervenções voltadas para a atenção psicossocial, promoção, prevenção de agravos e a recuperação da saúde mental dos adolescentes melhorando sua qualidade de vida e proporcionando um desenvolvimento mais saudável. Acrescentam-se as políticas de incentivo a uma maior vinculação do enfermeiro nas instituições escolares atuando de maneira eficaz e conjunta com os educadores na gestão das questões psicoemocionais dos adolescentes.

Sugere-se novas pesquisas com a Terapia Comunitária Integrativa voltadas para os adolescentes nos variados contextos 
em que estão inseridos e com diferentes números de rodas para avaliar se a diminuição dos sintomas de ansiedade depende do número de intervenções e se essa diminuição é progressiva e em relação as questões de gênero

É valida a replicação dessa investigação com adolescentes, em que haja um grupo controle, para a comparação com o grupo intervenção, em delineamentos de estudos do tipo ensaios clínicos controlados e randomizados.

\section{Referências}

Associação Brasileira de empresas de Pesquisa. (2016). Critério de classificação econômica Brasil. http://www.abep.org/criterio-brasil

Azevedo, E. B. de, Cordeiro, R. C., Costa, L. de F. P., Guerra, C. D. S., Filha, M. de O. F., \& Dias, M. D. (2013). Pesquisas brasileiras sobre Terapia Comunitária Integrativa. Rev. Bras. Pesq. Saúde, 15(3), 114-120. www.periodicos.ufes.br > article > download > 6333

Barbosa, G. A.; Barbosa, A. G., \& Gouveia, V. V. (2002). Transtorno de Ansiedade na infância e adolescência: um estudo de pre valência e validação de um instrumento (SCARED) de triagem. Infanto- Revista Neuropsiquiatria da Infância e Adolescência, 1(10), 34-47. https://www.researchgate.net/profile/Valdiney_Gouveia/publication/279193421_Transtorno_de_ansiedade_na_infancia_e_adolescencia_um_estudo_de_preva lencia_e_validacao_de_um_instrumento_SCARED_de_triagem/links/558db00a08ae15962d8947ed/Transtorno-de-ansiedade-na-infancia-e-adolescencia-umestudo-de-prevalencia-e-validacao-de-um-instrumento-SCARED-de-triagem.pdf

Barbosa, G. A; Barbosa, A. G. (2001). Apontamentos em psicopatologia infantil. João Pessoa: Idéia.

Barreto, A. de P. (2019). Terapia Comunitária: passo a passo. Fortaleza: Grafica LCR.

Beesdo, K., Knappe, S., \& Pine, D. S. (2009). Anxiety and Anxiety Disorders in Children and Adolescents: Developmental Issues and Implications for DSMV. Psychiatric Clinics of North America, 32(3), 483-524. https://doi.org/10.1016/j.psc.2009.06.002

Behrens, B., Swetlitz, C., Pine, D. S., \& Pagliaccio, D. (2019). The Screen for Child Anxiety Related Emotional Disorders (SCARED): Informant Discrepancy, Measurement Invariance, and Test-Retest Reliability. Child Psychiatry and Human Development, 50(3), 473-482. https://doi.org/10.1007/s10578-018-0854-0

Boaretto, J. P., Silva, M. Z. da, \& Martins, E. A. P. (2020). Ansiedade e depressão na universidade: contribuições da terapia comunitária integrativa. Temas Em Educ. e Saúd, 16(esp.1), 296-310. https://periodicos.fclar.unesp.br/tes/article/view/14309/9779

Braga, L. A. V., Dias, D., Filha, Ma. de O. F., Moraes, M. N. de, Araruna, M. H. M., \& Rocha, I. A. da. (2013). Terapia Comunitária e resiliência: história de mulheres. R. Pesq.: Cuid. Fundam. Online, 5(1), 3453-3471. https://doi.org/10.9789/2175-5361.2013v5n1p3453

Brum, E. H. M. de, \& Teixeira, M. A. P. (2020). Adaptação acadêmica de alunos de psicologia ao ensino superior: proposta de intervenção e avaliação ACA. Revista Psicologia \& Saberes, 9(14), 41-58. https://revistas.cesmac.edu.br/index.php/psicologia/article/view/1095

Carneiro, A. L. B., Ramos, S. C. de S., Ribeiro, R. M., Melo, C. de M. B. de, Melo, M. N. A. de, Aguiar, P. K. F. de, Monteiro, B. R., Ferreira, M. de L., \& Falcão, P. B. L. (2020). Terapia Comunitária Integrativa em Tempos de Pandemia: Encontros, encantos, (con)vivências e partilhas que transcendem as telas. Research, Society and Development, 9(11), 1-42. https://doi.org/http://dx.doi.org/10.33448/rsd-v9i11.9785

Cid, M. F. B., Squassoni, C. E., Gasparini, D. A., \& Fernandes, L. H. de O. (2019). Saúde mental infantil e contexto escolar: as percepções dos educadores. ProPosições, 30(e20170093), 1-24. https://www.scielo.br/scielo.php?script=sci_arttext\&pid=S0103-73072019000100509

Conroy, K., Curtiss, J. E., Barthel, A. L., Lubin, R., Wieman, S., Bui, E., Simon, N. M., \& Hofmann, S. G. (2019). Emotion Regulation Flexibility in Generalized Anxiety Disorder. Journal of Psychopathology and Behavioral Assessment, 42(1), 93-100. https://doi.org/10.1007/s10862-019-09773-8

Conselho Nacional de Saúde. Comitê Nacional de Ética em Pesquisa em Seres Humanos. Resolução n. 466, de 12 de dezembro de 2012 . Diretrizes e Normas Regulamentadoras de pesquisa desenvolvendo seres humanos. (2012). http://conselho.saude.gov.br/resolucoes/2012/Reso466.pdf

Dallalana, T. M. (2015). Terapia comunitária - do sofrimento difuso para a construção de significados: um modelo de acolhimento, aplicação e treinamento no campo da saúde mental. Temas Em Educação e Saúde, 11(0), 153-162. https://doi.org/10.26673/tes.v11i0.9174

Desousa, D. A., Salum, G. A., Isolan, L. R., \& Manfro, G. G. (2013). Sensitivity and specificity of the Screen for Child Anxiety Related Emotional Disorders (SCARED): A community-based study. Child Psychiatry and Human Development, 44, 391-399. https://doi.org/10.1007/s10578-012-0333-y

Erse, M. P. Q. de A., Simões, R. M. P., Façanha, J. D. N., Marques, L. A. F. A., Loureiro, C. R. E. C., Matos, M. E. T. S., \& Santos, J. C. P. (2016). Depressão em adolescentes em meio escolar: Projeto + Contigo. Revista de Enfermagem Referência. http://dx.doi.org/10.12707/RIV15026

Farinha, F. T., Bom, G. C., Kostrisch, L. M. Von, \& Prado, P. C. (2018). Correlação entre espiritualidade, religiosidade e qualidade de vida em adolescentes. Revista Bioética, 26(4), 567-573. https://doi.org/10.1590/1983-80422018264275

Gaete, A. E. G., \& Gois, M. J. S. de M. de. (2020). A terapia comunitária integrativa na abordagem da saúde mental na atenção primária: um relato de experiência. Temas Em Educ. e Saúde, 16(esp.1), 483-497. https://periodicos.fclar.unesp.br/tes/article/view/14314

Garcia, B. N., Tavares, A. V. M. T., \& Assunção, M. F. (2018). Terapia comunitária integrativa em saúde mental: por uma atenção dialógica, por um cuidado extramuros. Revista de Psicologia, 37(2), 183-188. http://www.periodicos.ufc.br/psicologiaufc/article/view/32944

Gomez, R., \& Vance, A. (2016). Children's Depression Inventory: Testing Measurement Invariance for the Hierarchical Factor Model Across Children and Adolescents in a Clinic-Referred Sample. Journal of Childhood \& Developmental Disorders, 2(3), 1-8. https://doi.org/10.4172/2472-1786.100032 
Grolli, V., Wagner, M. F., \& Dalbosco, S. N. P. (2017). Sintomas Depressivos e de Ansiedade em Adolescentes do Ensino Médio. Revista de Psicologia Da IMED, 9(1), 87. https://doi.org/10.18256/2175-5027.2017.v9i1.2123

Hofmann, S. G., Sawyer, A. T., Fang, A., \& Asnaani, A. (2012). Emotion dysregulation model of mood and anxiety disorders. Depression and Anxiety, 29(5), 409-416. https://doi.org/10.1002/da.21888

Ingul, J. M., \& Nordahl, H. M. (2013). Anxiety as a risk factor for school absenteeism: What differentiates anxious school attenders from non-attenders? Annals of General Psychiatry, 12(1), 1-9. https://doi.org/10.1186/1744-859x-12-25

Inoue, T. M., \& Vecina, M. V. A. (2017). Espiritualidade e/ou religiosidade e saúde: uma revisão de literatura. J Health Sci Inst., 35(2), 127-130. http://seer.pucgoias.edu.br/index.php/caminhos/article/view/6939

Kent, B. V., Stroope, S., Kanaya, A. M., Zhang, Y., Kandula, N. R., \& Shields, A. E. (2020). Private religion/spirituality, self-rated health, and mental health among US South Asians. Quality of Life Research, 29(2), 495-504. https://doi.org/10.1007/s11136-019-02321-7

Kim, S., \& Esquivel, G. B. (2011). Adolescent spirituality and resilience: theory, research, and educational practices. Psychology in the Schools, 48(7), 755765. https://doi.org/10.1002/pits

Lemes, A. G., Nascimento, V. F. do, Rocha, E. M. da, Almeida, M. A. S. O., Volpato, R. J., \& Luis, M. A. V. (2020). Terapia Comunitária como cuidado complementar a usuários de drogas e suas contribuições sobre a ansiedade e a depressão. Escola Anna Nery, 24(3), 1-8. https://doi.org/10.1590/2177-9465-ean2019-0321

Lutterbach, M. G. C. (2017). A contribuição da Terapia Comunitária Integrativa (TCI) na produção do cuidado e formação em saúde na atenção psicossocial. Dissertação de Mestrado, Universidade Federal Fluminense, Rio de Janeiro, RJ, Brasil. https://app.uff.br/riuff/bitstream/1/4283/1/Marise Gama Correa Lutterbach.pdf

Madasu, S., Malhotra, S., Kant, S., Sagar, R., Mishra, A. K., Misra, P., \& Ahamed, F. (2019). Prevalence and determinants of anxiety disorders among adolescents in a rural community from northern India. Asian Journal of Psychiatry, 43(33), 137-142. https://doi.org/10.1016/j.ajp.2019.05.009

Malinakova, K., Kopcakova, J., Madarasova Geckova, A., van Dijk, J. P., Furstova, J., Kalman, M., Tavel, P., \& Reijneveld, S. A. (2019). "I am spiritual, but not religious": Does one without the other protect against adolescent health-risk behaviour? International Journal of Public Health, 64(1), 115-124. https://doi.org/10.1007/s00038-018-1116-4

Miller, K. A., Ritt-olson, A., Wojcik, K. Y., Freyer, D. R., Ramirez, C. N., Hamilton, A. S., \& Milam, J. E. (2017). Supporting long-term follow-up of young adult survivors of childhood cancer : Correlates of healthcare self-efficacy. Pediatr Blood Cancer, 64(2), 358-363. https://doi.org/10.1002 / pbc.26209

Moura, S. G. de, Filha, M. de O. F., Cordeiro, R. C., Braga, L. A. V., \& Monteiro, C. Q. A. (2012). A experiência da Terapia Comunitária em diferentes instituições e contextos populacionais. Revista da Universidade Vale do Rio Verde, 10(1), 329-338. http://dx.doi.org/10.5892/ruvrv.2012.101.329338

Moura, S. G. de, Filha, M. de O. F., Moreira, M. A. S. P., Simpson, C. A., Tura, L. F. R., \& Silva, A. O. (2017). Representações sociais sobre terapia comunitária integrativa construídas por idosos. Revista Gaúcha de Enfermagem, 38(2), e55067. https://doi.org/10.1590/1983-1447.2017.02.55067

Mourão, L. F., Oliveira, L. B. de, Marques, A. D. B., Branco, J. G. de O., Guimaraes, M. do S. de O., \& Nery, I. S. (2016). Terapia comunitária como novo recurso da prática do cuidado: revisão integrativa. Sanare, 15(2), 129-135. https://sanare.emnuvens.com.br/sanare/article/view/1047/593

Nunes, C. K., Silva, A. B. da, Kantorski, L. P., Coimbra, V. C. C., \& Olschowsky, A. (2020). Cuidado intersetorial em saúde mental na infância e adolescência: para além da instituição saúde. Revista de Pesquisa Cuidado é Fundamental Online, 12, 233-238. https://doi.org/10.9789/2175-5361.rpcfo.v12.8277

Oliveira Cezário, P. F., Lins de Araújo, L., Barbosa Pereira, T. L., Braga Teixeira, O. F., Brasil Abrantes, A. W., de Sousa Andrade, E., Gonçalves Almeida, N., Silva Sobreira, M. V., Damasceno Moraes, J. S., Oliveira Cezário, P., do Nascimento Andrade Feitosa, A., de Oliveira Maciel Freitas, A. E., de Abreu Andrade Moreira, S. C., Esmeraldo Neves, F. K., \& Bertino Véras, G. C. (2015). Integrative Community Therapy and its benefits for primary care: an integrative review. International Archives of Medicine, 8(267), 1-9. https://doi.org/10.3823/1866

Oliveira, S. M. De, Junior, J. O. M., Junior, S. V. D. S., Dias, M. D., Fernandes, M. D. G. M., \& Filha, M. D. O. (2017). Rodas de terapia comunitária: construindo espaços terapêuticos para idosos em comunidades quilombolas. Rev Enferm UFSM, 7(4), 712-724. https://doi.org/10.5902/2179769220299

Organização Mundial da Saúde. (2017). Global Accelerated Action for the Health of Adolescents. Guidance to Support Country Implementation Global. www.who.int/life-course

Organização Pan-Americana da Saúde. $\quad$ (2018). Saúde $\quad$ Mental dos adolescentes. https://www.paho.org/bra/index.php?option=com_content\&view=article\&id=5779:folha-informativa-saude-mental-dos-adolescentes\&Itemid=839\%3E

Orellana, J. D. Y., Ribeiro, M. R. C., Barbieri, M. A., Saraiva, M. da C., Cardoso, V. C., Bettiol, H., Silva, A. A. M. da, Barros, F. C., Gonçalves, H., Wehrmeister, F. C., Menezes, A. M. B., Del-Ben, C. M., \& Horta, B. L. (2020). Transtornos mentais em adolescentes, jovens e adultos do Consórcio de Coortes de Nascimento brasileiras RPS (Ribeirão Preto, Pelotas e São Luís). Cadernos de Saúde Publica, 36(2), e00154319. https://doi.org/10.1590/0102-311X00154319

Patias, N. D., Machado, W. D. L., Bandeira, D. R., \& Dell'Aglio, D. D. (2016). Depression Anxiety and Stress Scale (DASS-21) - Short Form: Adaptação e Validação para Adolescentes Brasileiros. Psico-USF, 21(3), 459-469. https://doi.org/10.1590/1413-82712016210302

Pereira A.S., Shitsuka D.M., Parreira F.J., \& Shitsuka R. (2018). Metodologia da pesquisa científica. Santa Maria: UAB/NTE/UFSM. : em: https://repositorio.ufsm.br/bitstream/handle/1/15824/Lic_Computacao_Metodologia-Pesquisa-Cientifica.pdf?sequence=1

Polit, D.F.; Beck, C. T. (2018). Fundamentos de Pesquisa em Enfermagem - Avaliação de Evidências para a Prática da Enfermagem. Porto Alegre: Artmed.

Portaria no 849, de 27 de março de 2017 (2017). Inclui a Arteterapia, Ayurveda, Biodança, Dança Circular, Meditação, Musicoterapia, Naturopatia, Osteopatia, Quiropraxia, Reflexoterapia, Reiki, Shantala, Terapia Comunitária Integrativa e Yoga à PNPIC. Março de 2017. http://bvsms.saude.gov.br/bvs/saudelegis/gm/2017/prt0849_28_03_2017.html 
Research, Society and Development, v. 10, n. 3, e5010312986, 2021

(CC BY 4.0) | ISSN 2525-3409 | DOI: http://dx.doi.org/10.33448/rsd-v10i3.12986

Reis, M. L. de A., \& Grandesso, M. (2014). O significado da capacitação em Terapia Comunitária Integrativa na vida dos terapeutas comunitários. Temas Em Educação e Saúde, 10, 89-116. https://doi.org/DOI: https://doi.org/10.26673/tes.v10i0.9617

Runyon, K., Chesnut, S. R., \& Burley, H. (2018). Screening for childhood anxiety : A meta-analysis of the screen for child anxiety related emotional disorders. Journal of Affective Disorders, 240 (June), 220-229. https://doi.org/10.1016/j.jad.2018.07.049

Silva, A. L. da, Reis, E. C. dos, \& Greinert, B. R. M. (2016). Atuação do enfermeiro na promoção da saúde escolar. Anais do II Congresso Brasileiro Interdisciplinar de Promoção da Saúde. Universidade de Santa Cruz do Sul. https://online.unisc.br/acadnet/anais/index.php/CBIPS/article/view/16087/3974

Soares, F. C., Hardman, C. M., Rangel Junior, J. F. B., Bezerra, J., Petribú, K., Mota, J., de Barros, M. V. G., \& Lima, R. A. (2020). Secular trends in suicidal ideation and associated factors among adolescents. Brazilian Journal of Psychiatry, 42(5), 475-480. https://doi.org/10.1590/1516-4446-2019-0783

Tardivo, L. S. de L. P. C., Rosa, H. R., Ferreira, L. S., Chaves, G., \& P. Junior, A. A. (2019). Autolesão em adolescentes, depressão e ansiedade: um estudo compreensivo. Bol. - Acad. Paul. Psicol., 39 (97), 157-169. https://doi.org/10.5935/2176-3038.20190015

Tavares, B. F., Béria, J. U., \& Lima, M. S. de. (2004). Fatores associados ao uso de drogas entre adolescentes escolares. Rev Saúde Pública, 38(6), 787-796. www.fsp.usp.br/rsp

Teixeira, L. A., Freitas, R. J. M. de, Moura, N. A. de, \& Monteiro, A. R. M. (2020). Necessidades De Saúde Mental De Adolescentes E Os Cuidados De Enfermagem : Revisão Integrativa. Texto e Contexto Enfermagem, 29, 1-15. https://doi.org/http://dx.doi.org/10.1590/1980-265X-TCE-2018-0424 1/15

Thiengo, P. C. D. S., Gomes, A. M. T., Das Mercês, M. C. C., Couto, P. L. S., França, L. C. M., \& Da Silva, A. N. (2019). Espiritualidade e Religiosidade no Cuidado em Saúde: Revisão Integrativa. Cogitare Enfermagem, 24. https://doi.org/10.5380/ce.v24i0.58692

Tomé, G., Matos, M. G. de, Gomes, P., Camacho, I., \& Gaspar, T. (2017). Promoção da saúde mental nas escolas - Projeto ES’COOL. Revista de Psicologia da Criança e do Adolescente, 8(1), 173-184. http://revistas.lis.ulusiada.pt/index.php/rpca/article/view/2485

Turpyn, C. C., Chaplin, T. M., Cook, E. C., \& Martelli, A. M. (2015). A Person-Centered approach to adolescent emotion regulation: associations with psychopathology and parenting. Physiology \& Behavior, 136(1-16), 1-26. https://doi.org/10.1016/j.physbeh.2017.03.040

Wiens, K., Bhattarai, A., Pedram, P., Dores, A., Williams, J., Bulloch, A., \& Patten, S. (2020). A growing need for youth mental health services in Canada: Examining trends in youth mental health from 2011 to 2018. Epidemiology and Psychiatric Sciences, 29(e115), 1-9. https://doi.org/10.1017/S2045796020000281

Zem Igeski, T. P., Silva, L. P. da, Silva, D. B. da, \& Silva, M. Z. da. (2020). Análise da efetividade da terapia comunitária integrativa na saúde biopsicossocial de diferentes populações: uma revisão integrativa. Temas Em Educ. e Saúde, 16(esp.1), 271-285. https://doi.org/10.26673/tes.v16iesp.1.13737

Zidkova, R., Glogar, P., Solcova, I. P., Van Dijk, J. P., Kalman, M., Tavel, P., \& Malinakova, K. (2020). Spirituality, religious attendance and health complaints in czech adolescents. International Journal of Environmental Research and Public Health, 17(7), 1-9. https://doi.org/10.3390/ijerph17072339 